\title{
EVALUACIÓN DE RIESGOS DE COLISIÓN Y ELECTROCUCIÓN DE LOS TENDIDOS ELÉCTRICOS DE LAS ZEPAs DEL ÁMBITO DE APLICACIÓN DEL PLAN DE RECUPERACIÓN DEL QUEBRANTAHUESOS (Gypaetus barbatus) EN ARAGÓN
}

\author{
J. A. GIL \\ Fundación para la Conservación del Quebrantahuesos (FCQ). \\ Plaza San Pedro Nolasco, 1, 4-F. 50001 Zaragoza \\ fcq@quebrantahuesos.org
}

\begin{abstract}
In the last 30 years (1979-2008) 18 cases of mortality of Bearded Vulture (Gypaetus barbatus) by electrocution and collision with electrical lines in the Pyrenees have been registered (Spain-France). The results of the evaluation on the electrocution and airmisses of the electrical layings of the Important Bird Areas (IBA) of the scope of application of the Plan of Recovery of the Bearded Vulture in Aragon, propose the correction of 138 electrical lines, 1127 kilometers and 4804 supports.
\end{abstract}

Keywords: IBA, Bearded Vulture, electrical lines, electrocution and collision.

RESUMEN.- En los últimos 30 años (1979-2008) se han registrado 18 casos de mortalidad de quebrantahuesos (Gypaetus barbatus) por electrocución y colisión con líneas eléctricas en los Pirineos (España-Francia). Los resultados de la evaluación sobre los riesgos de colisión y electrocución de los tendidos eléctricos de las Zonas de Especial Protección para las Aves (ZEPAs) del ámbito de aplicación del Plan de Recuperación del Quebrantahuesos en Aragón proponen la corrección de 138 líneas eléctricas, 1127 kilómetros y 4804 apoyos.

Palabras clave: ZEPAs, quebrantahuesos, tendidos eléctricos, medidas anticolisión y antielectrocución. 


\section{Introducción}

Estudios sobre la dinámica poblacional, dispersión juvenil y uso del espacio de las aves han demostrado que la electrocución y colisión en tendidos eléctricos es una de las principales causas de mortalidad no natural de especies amenazadas: águila imperial (Aquila adalberti), quebrantahuesos, buitre negro (Aegypius monachus), etc. (FERRER \& NEGRO, 1992; BEVANGER, 1998).

A diferencia de otros problemas ambientales, existen técnicas sencillas y eficaces para eliminar los riesgos de electrocución y colisión de las aves (balizamiento, aislamiento de conductores) (FERNÁNDEZ \& AZCONA, 2002).

En los últimos 30 años (1979-2008) se han registrado 18 casos de mortalidad de quebrantahuesos por electrocución $(33,3 \%)$ y colisión $(66,7 \%)$ con líneas eléctricas en los Pirineos (España-Francia), siendo la tercera causa de mortalidad no natural de la especie en Europa (MARGALIDA et al., 2008) (ver tabla 1).

La incidencia de los tendidos eléctricos en la avifauna empezó a ser considerada en varios países a mediados de los años setenta (Holanda y Estados Unidos). En España los primeros trabajos de importancia no se realizaron hasta los años 80 del siglo XX, siendo el estudio más conocido el del águila imperial. La información acerca de este problema en Aragón ha ido aumentando progresivamente durante estos últimos años. Al principio tan solo se disponía de registros aislados de accidentes de aves recogidos en el Centro de Recuperación de Fauna Silvestre de la Alfranca (Gobierno de Aragón-GA-). Ante la sospecha de que las líneas eléctricas pudieran estar provocando accidentes preocupantes en algunas aves de Aragón, el GA realizó varios estudios sobre la incidencia de los tendidos en las aves: águila perdicera (Hieraetus fasciatus), buitre leonado (Gyps fulvus), grulla común (Grus grus) y avutarda (Otis tarda) (PELAYO \& SAMPIETRO, 2000).

En 1994 se aprueba el Plan de Recuperación del Quebrantahuesos en Aragón (Decreto 184/1994). El Plan estableció necesario la realización de un informe previo favorable en aquellos tendidos eléctricos de nueva instalación o que se tuvieran que reformar y que afectaran a Áreas Críticas de la especie. Gracias a ello durante el periodo comprendido entre 1997 y 2005 técnicos del GA y de la FCQ realizaron informes sobre tendidos eléctricos, en los que incorporaron medidas anticolisión y electrocución en 160 líneas eléctricas.

En 2002 se firmaron dos Convenios Marcos de Colaboración entre GA y ERZ-ENDESA: uno para el estudio y evaluación de los riesgos de colisión y electrocución de de los tendidos eléctricos de las ZEPAs del ámbito de aplicación del Plan de Recuperación del Quebrantahuesos en Aragón y otro para reducir y eliminar riesgos. El primero fue desarrollado por la FCQ entre los 
EVALUACIÓN DE RIESGOS DE COLISIÓN Y ELECTROCUCIÓN DE LOS TENDIDOS ELÉCTRICOS...

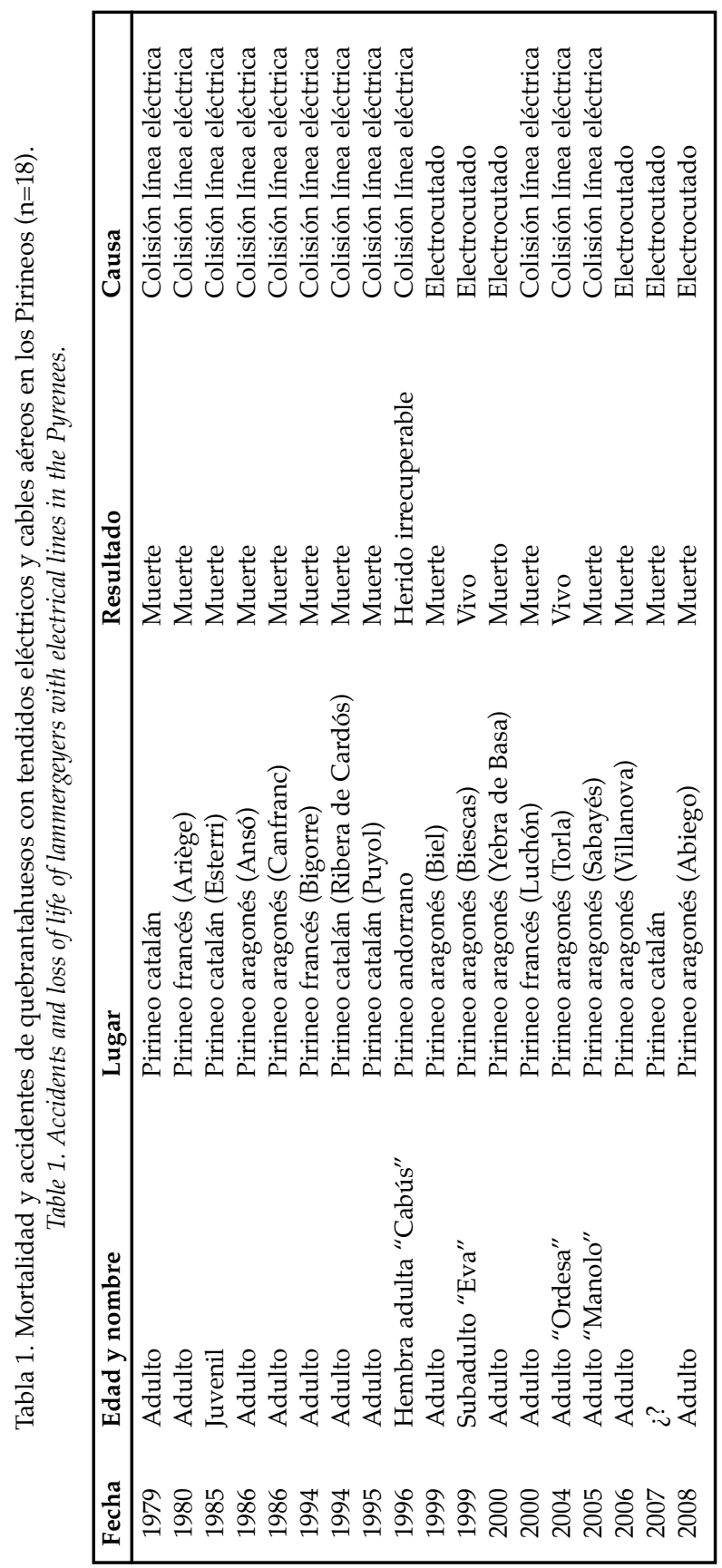


años 2003 y 2005 y financiado por ERZ-ENDESA. Sus objetivos fueron los siguientes:

- Describir los modelos de apoyos y armados de los tendidos eléctricos.

- Cartografiar las líneas eléctricas, incluyendo mapa de peligrosidad.

- Identificar los apoyos y trazados susceptibles de ocasionar accidentes.

- Proponer la corrección de tendidos eléctricos, con el fin de eliminar o mitigar el riesgo de electrocución y colisión.

- Valorar la peligrosidad en función las características, ubicación y mortalidad.

- Elaborar una base de datos compatible con el sistema SIG.

- Estimar el coste económico de las correcciones.

\section{Material y métodos}

El área de trabajo estaba situada en el Pirineo Central (Aragón-España), concretamente en 15 ZEPAs $(3180 \mathrm{~km}$ ) del ámbito de aplicación del Plan de Recuperación del Quebrantahuesos en Aragón (14.630km²) (mapa 1 y 2 y tabla 2). En estas ZEPAs se localizan el $87,5 \%$ de las unidades reproductoras de quebrantahuesos de Aragón, según el inventario de la población reproductora de 2004 (GIL \& ALCANTARA, 2004).

A continuación se describe la metodología empleada:

- Recopilación de la cartografía digital del área de estudio (ERZ-ENDESA).

- Preparación e impresión de la ficha y la cartografía de campo (1:50.000).

- Formación del equipo de trabajo (tres personas).

- Prospección de campo y recogida de información (se realiza un catálogo de modelos de apoyos en el área de estudio: 124 apoyos diferentes). Se recorren tanto los tendidos del interior de la ZEPA como los situados en un perímetro cercano.

- Redacción de informes técnicos para la corrección de las líneas eléctricas siguiendo criterios de eficacia y rentabilidad coste/beneficio (JANSS \& FERRER, 1999; FERNÁNDEZ \& AZCONA, 2002). La peligrosidad del tendido viene determinada por el tipo de apoyo o por el trazado. En los informes se incluye la siguiente información:

- Características de las líneas eléctricas (tensión nominal, propiedad, longitud, tipo de modelo de apoyo).

- Medidas anti-electrocución: aislamiento con material termoretráctil de los elementos de tensión, modificación de armados sustituyendo 

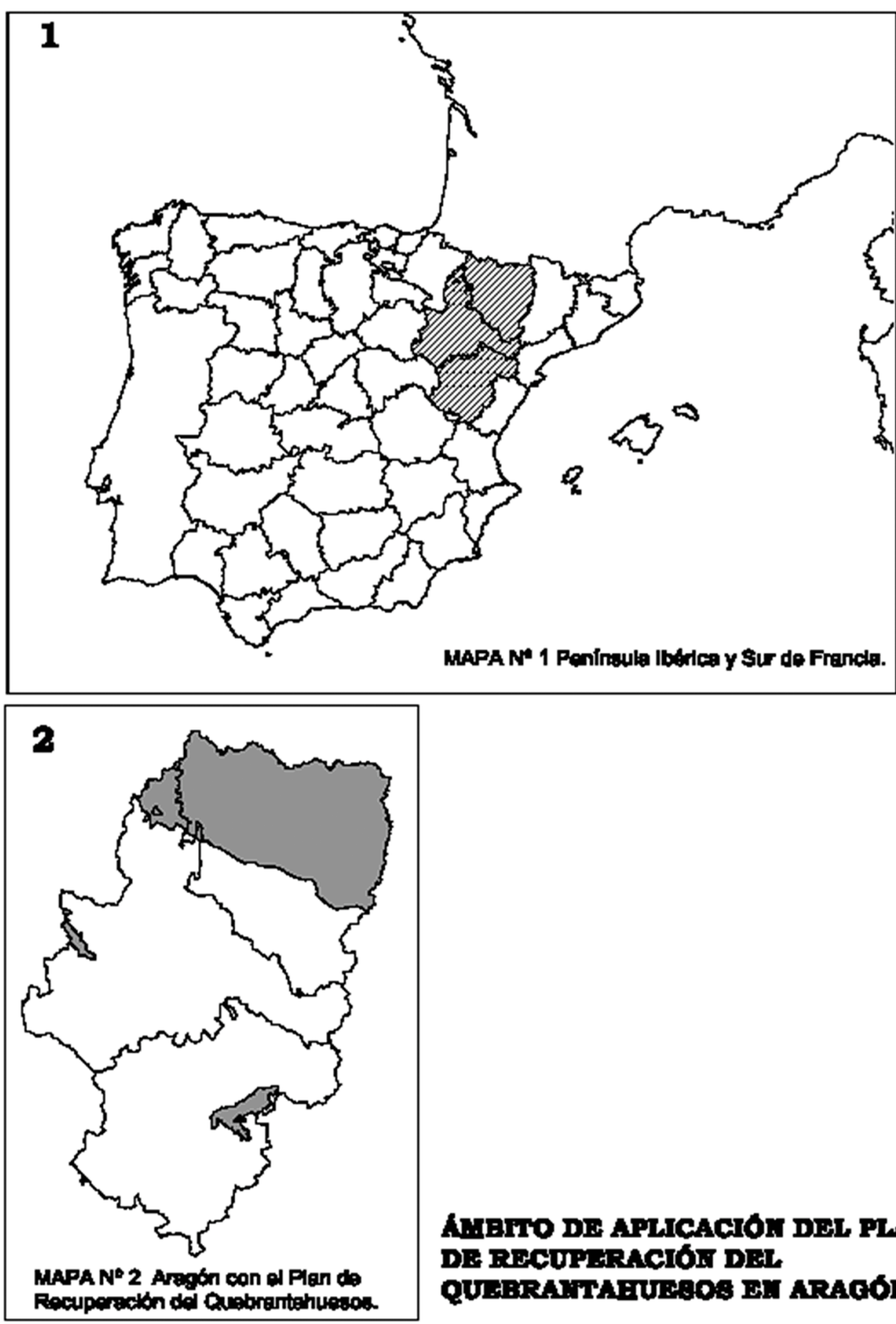

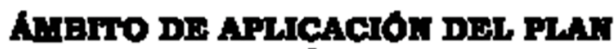

Figura 1. Ámbito de aplicación del Plan de Recuperación del Quebrantahuesos en Aragón. Figure 1. Area of lammergeyer conservation plan in Aragón. 
Tabla 2. Zonas de aplicación del Plan de Recuperación del Quebrantahuesos en Aragón. Table 2. Areas of lammergeyer conservation plan in Aragón.

\begin{tabular}{|cc|}
\hline ZEPAs & Ha. \\
\hline Los Valles & 31982 \\
Collarada-Ibón de Ip & 3456 \\
Viñamala & 25302 \\
Ordesa y Monte Perdido & 15797 \\
Alto Cinca & 14708 \\
Posets-Maladeta & 34216 \\
Salvatierra-Foces de Fago-Biniés-Bco. del Infierno & 2578 \\
Sierras de Leyre y Orba & 5892 \\
San Juan de la Peña y Peña Oroel & 6149 \\
Sierras de Santo Domingo, Caballera y río Onsella & 35747 \\
Cancias-Silves & 7810 \\
Sierra y Cañones de Guara & 81412 \\
Cotiella-Sierra Ferrera & 25331 \\
El Turbón y Sierra de Sis & 24396 \\
Sierra de Mongay & 3226 \\
\hline TOTAL & 318002 \\
\hline
\end{tabular}

aislamientos rígidos a suspensión y reinstalación de los puentes flojos en posición suspendida.

- Medidas anti-colisión: señalización de los vanos con balizas salvapájaros en los tramos peligrosos.

- Estimación de presupuestos según precios ERZ-ENDESA del año 2002.

- Estimación del índice de accidentalidad: $\mathrm{n}^{\circ}$ de aves electrocutadas $\mathrm{x}$ $100 / \mathrm{n}^{\circ}$ de apoyos $\mathrm{X} \mathrm{n}^{\circ}$ de visitas (PELAYO \& SAMPIETRO, 2000).

- Cartografía 1:20.000, 1:50.000 y 1:150.000.

\section{Resultados}

En el ámbito de aplicación del Plan existen 4655,21 kilómetros de tendidos eléctricos $\left(0,318 \mathrm{~km}\right.$. de líneas $\left./ \mathrm{km}^{2}\right)$. Las 15 ZEPAs del ámbito del Plan poseen en su interior 424,74 kilómetros de líneas eléctricas, el 9,1\% de todos los tendidos eléctricos del ámbito del Plan $(0,133 \mathrm{~km}$. de líneas $/ \mathrm{km} 2)$. La ZEPA con menor densidad de líneas eléctricas fue Posets-Maladeta $(0,002 \mathrm{~km}$. de líneas $\left./ \mathrm{km}^{2}\right)$ y la de mayor densidad San Juan de la Peña y Peña Oroel $(0,340$ $\mathrm{km}$. de líneas $/ \mathrm{km} 2$ ). La única ZEPA que no posee tendidos eléctricos fue la de 
Collarada-Ibón de Ip. Se propone la corrección de 138 líneas eléctricas (1127 kilómetros). La ZEPA con mayor número de tendidos eléctricos y kilómetros a corregir fue la Sierra y Cañones de Guara $(n=41 / n=219 \mathrm{~km}$.). Se propone la corrección de 4804 apoyos. La ZEPA con mayor número de apoyos a corregir fue la Sierra Sto. Domingo y Caballera $(\mathrm{n}=880)$. Se propone la señalización de 2031 vanos. La ZEPA con mayor número de vanos a corregir fue la de TurbónSierra de Sis $(\mathrm{n}=260)$. El presupuesto estimado para todas las correcciones fue de 2.998.000€. Se revisaron 687 apoyos, obteniéndose un índice de accidentalidad prácticamente nulo.

\subsection{Medidas de gestión}

Gracias a los Convenios Marco de Colaboración entre el GA, ERZ-ENDESA y REE, las empresas eléctricas se comprometieron a corregir las instalaciones de su propiedad. Para costear parte de los gastos de la inversión se contó con el apoyo financiero de un programa LIFE-Naturaleza (2004-2007). Entre el 2002-2006 se corrigieron 63 instalaciones eléctricas, remodelando 908 apoyos en $163 \mathrm{~km}$. de tendido y señalizando 750 vanos y $150 \mathrm{~km}$. de línea. En el marco de este programa se corrigieron 19 tendidos eléctricos del ámbito de aplicación del Plan. Por último en 2005 el GA aprobó normativa electrotécnica (Decreto 34/2005), cuyo fin es el de asegurar una serie de prescripciones técnicas en las líneas eléctricas de nueva instalación, para evitar accidentes entre las aves.

\section{Conclusiones}

La tercera causa de mortalidad no natural del quebrantahuesos en los Pirineos es la colisión y electrocución con tendidos eléctricos. Para eliminar este problema ambiental es necesario evaluar los riesgos de estas infraestructuras, aprobar normativa electrotécnica y realizar acciones que reduzcan la mortalidad (aislamientos de conductores, balizamiento, etc.) en colaboración con las compañías eléctricas implicadas. En el ámbito de aplicación del Plan de Recuperación del Quebrantahuesos en Aragón se han realizado durante los últimos años considerables esfuerzos en la corrección de tendidos eléctricos peligrosos para las aves, sin embargo se tendrá que seguir realizando una importante inversión personal y económica con el fin de reducir y evitar está problemática medioambiental. 


\section{Agradecimientos}

ERZ-ENDESA, REE, Departamento de Medio Ambiente del Gobierno de Aragón, LIFE, Interreg IIIA, Parque Nacional de Ordesa y Mte. Perdido, Parque Natural Posets-Maladeta, Parque Natural de la Sierra y los Cañones de Guara, SEO/BirdLife y ANSAR. Muy especialmente: J.C. Albero, M. Rollan. L. Tirado, C. Pérez, J. Losada, J. Guiral, J. Insausti, M. Alcantara, F. Hernández, J. Fumanal, J. Jabal, C. Fernández. P. Azkona, E. Pelayo, J. Sampietro, R. Antor, G. Báguena, L. Lorente, O. Díez y E. Palacios.

\section{Referencias}

BEVANGER, K. (1998). Biological and conservation aspects of birds mortality causes by electricity power lines: a review. Biol. Conserv., 86: 67-76.

FERNÁNDEZ, C. \& AZCONA, P. (2002). Tendidos eléctricos y medio ambiente en Navarra. Ed. Gobierno de Navarra. Medio Ambiente.

FERRER, M. \& NEGRO, J.J. (1992). Tendidos eléctricos y conservación de aves en España. Ardeola, 39: 23-28.

GIL. J.A. \& ALCANTARA, M. (2004). Informe anual 2004. Programa técnico: estudio y conservación del quebrantahuesos (Gypaetus barbatus) en Aragón. Departamento de Medio Ambiente del Gobierno de Aragón. Informe inedito.

JANSS, G.F. \& FERRER, M. (1999). La electrocución de aves en los apoyos de los tendidos eléctricos: Experiencias europeas (pag: 155-177) en FERRER, M. \& JANSS, G.F. (eds.). Aves y líneas eléctricas: colisión, electrocución y nidificación. Ed. Quercus.

MARGALIDA, A., HEREDIA, R., RAZIN, M. \& HERNÁNDEZ, M. (2008). Sources of variation in mortality of the Bearded Vulture Gypaetus barbatus in Europe. Bird Conservation Internacional, 18: 1-10.

PELAYO, E. \& SAMPIETRO, J. (2000). Incidencia de los tendidos eléctricos sobre aves sensibles en Aragón. Ed. Consejo de Protección de la Naturaleza de Aragón. Serie Investigación. No 19. 